\title{
A Review on Reliability and Validity of CRISPR/Cas9 Technology for Gene Editing
}

\author{
Bishnu Dev Das ${ }^{1}$ and Niroj Paude ${ }^{1,2,3, *}$ \\ ${ }^{1}$ Department of Botany, Mahendra Morang Aadarsh Multiple Campus Biratnagar (Tribhuvan University), Nepal, ${ }^{2}$ Department of Applied \\ Plant Science, Kangwon National University, Chuncheon 24341, Republic of Korea. ${ }^{3}$ National Institute of Horticultural and Herbal \\ Science, Rural Development Administration, Wanju 55365, Republic of Korea
}

Received: July 24, 2020; Revised: October 3, 2020; Accepted: October 18, 2020

\begin{abstract}
Genome engineering is one of the worldwide fast growing field of biotechnology which involves designed programmable DNA-binding nucleases such as homing endonucleases, zinc finger nucleases (ZFNs), transcriptions activator like effector nucleases (TALENs) and clustered regularly interspaced short palindromic repeats (CRISPR)/Cas9 (CRISPR-associated 9) nucleases. These technologies utilize manipulated nucleases which are the complex of sequence-specific DNA binding domains and nonspecific DNA cleavage modules. CRISPR)/Cas9 technology lets scientists accurately cut and paste genes into DNA which can be applied to edit the individual gene or even entire chromosomes from an organism at any point in its development, become a magical tool due to its simplicity. Here we review the four basic pieces of information on the genome editing technologies with their reliability and discuss the applications and their therapeutic potential as well as future prospects.
\end{abstract}

Key words: Gene editing, CRISPR/Cas9 technology, endonucleases mechanism, transcription.

\section{Introduction}

In 1905, in a letter to his colleague Adam Sedgwick, the English biologist William Bateson used the word 'genetics' to designate 'the science of heredity and variation’ (Gayon, 2016). Onwards 1970s, Genome Editing (GE) technologies establish a new revolution in modern research in genetics or biology. The recent advance technology CRISPR/Cas9 technology are used systematic interrogation of mammalian function of genome (Hsu et al., 2014) (Fig. 1). In life science research, genome editing can delete, insert, and modify the DNA sequences of cells that enable the function of specific genes. The biotechnologies used for gene editing are, (1) homing endonucleases or meganucleases), (2) zinc-finger nucleases (ZFNs), (3) transcription activator-like effector nucleases (TALENs and (4) clustered regularly interspaced short palindromic repeats (CRISPR)-CRISPR-associated protein 9 (Cas9). Out of these fours, CRISPRCas9 and TALENs are new genomic sequences that have driven a revolution in genome editing that has accelerated scientific breakthroughs and discoveries in multiple practices such as synthetic biology, human gene therapy, disease modeling, drug discovery, neuroscience, and the agricultural sciences (Gaj et al., 2016).

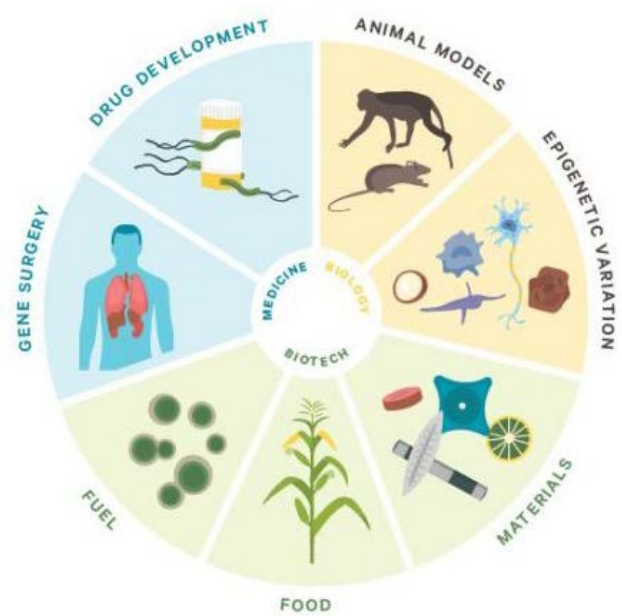

Figure 1. Applications of Genome Engineering (Hsu et al., 2014)

\section{Homing endonucleases or meganucleases}

Endonuclease enzymes are involved in genomic modification, rearrangement, protection, and repair. They specify at least nine orders of magnitude, ranging from nonspecific degradative enzymes up to a variety of genespecific endonucleases, and most specific enzymes are called homing endonucleases that produce double-strand breaks at individual loci in their host genomes and drive site-specific gene conversion events. The first observation of homing dates to experiments conducted at the Pasteur Institute in the early 1970s, and investigators noted the dominant inheritance of a genetic marker, termed 'omega,'

\footnotetext{
${ }^{*}$ Corresponding author e-mail: nirojjirauna@gmail.com.
} 
during yeast mating experiments (mitochondrial genes are passed on biparentally in such studies and are thus related to Mendelian laws of inheritance). Omega was located inside the mitochondrial gene that encodes the large ribosomal RNA subunit (LSrRNA) (Bolotin et al., 1971) and was inherited about $100 \%$ frequency in experimental processes of homozygous 'omega-plus' and “'omegaminus” yeast strains (Netter et al., 1974). In subsequent experiments, omega was found to correspond to an intervening sequence (recognized as a self-splicing group I intron) (Faye et al., 1979).

Mega-nuclease two enzyme such as intron endonuclease and intein endonuclease. Homing endonucleases (meganucleases) are the final member of the targeted nuclease family which have been reviewed at length elsewhere (Silva et al., 2011; Stoddard, 2014). But endonuclease bind amino acid present of the enzymes that recognize and cleave long DNA sequence (Figure 2).

The intron is driven by a site-specific endonuclease (now termed I-SceI) that is encoded by an open reading frame harbored within the intron sequence (Jacquier and Dujon, 1985), which generates a DNA double-strand break within a long DNA target sequence in the LSrRNA gene that contains the eventual intron insertion site. Improvement through homologous recombination using the intron containing allele as a corrective template leads to a duplication of the intron and its endonuclease gene into the target site (Figure 3). Homing is a process in which microbial self-splicing intervening sequences group I or group II introns or inteins are specifically duplicated into recipient alleles of their host gene that lack such a sequence (Chevalier and Stoddard, 2001).

Families and Structures of homing endonucleases are universal and are found in microbes from all biological kingdoms, corresponding phage and viruses. Despite the closeness and the frequent symbiotic relationship between multicellular eukaryotes and various microbial species, no examples have been reported of homing endonuclease genes within genomes of those more complex organisms. There are five different families of homing endonucleases recognized and initially associated with a specific biological host range (Stoddard, 2005).

\section{Zinc-finger nucleases (ZFNs)}

The recent advancements in genome editing include site-specific nucleases, usage of which for genome editing began with the arrival of zinc-finger nucleases (ZFNs) in 2002. The ZFNs were the first specific protein reagents that revolutionized the field of genome manipulation. ZFNs are DNA binding domains and specifically recognize three base pairs at the target site (Rai et al., 2019).ZFNs are formed by the combination between $\mathrm{Cys}_{2}$ $\mathrm{His}_{2}$ zinc-finger protein and the cleavage domain of the FokI restriction endonuclease (Kim et al. 1996) that are the first targeted gene to achieve universal use (Urnov et al., 2010).ZFNs behaves as dimers, with each monomer observing a specific "half site" sequence typically nine to 18 base pairs (bps) of DNA via the zinc-finger DNAbinding domain (Fig. 2).

The FokI cleavage domain regulates the dimerization of ZFN which cuts DNA within a five to seven-bp spacer sequence that separates two flanking zinc-finger binding sites (Smith et al., 2000). Primarily, each ZFN is made up of three or four zinc-finger domains, with each individual domain composed of 30 amino acid residues that are organized in a bba (beta beta alpha) motif (Pavletich and Pabo, 1991). The residues that facilitate DNA recognition are located within the a-helical domain and typically interact with three bps of DNA, with occasional overlap from an adjacent domain (Wolfe et al., 2000). Using methods such as phage display (Wu et al., 1995), a large number of zinc finger domains recognizing distinct DNA triplets have been identified (Dreier et al., 2005). These domains can be merged in tandem using a canonical linker peptide to produce polydactyl zinc-finger proteins that can target a wide range of possible DNA sequences (Kim et al., 2009). Besides this "modular assembly" approach to zinc-finger construction, selection-based methods for constructing zinc-finger proteins have also been reported (Magnenat et al., 2004), including those that consider context-dependent interactions between adjacent zincfinger domains, such as oligomerized pool engineering (OPEN) (Maeder et al., 2008). Moreover, particular sets of validated two-finger, zinc-finger modules have been used to gather zinc finger arrays (Kim et al., 2009; Bhakta et al., 2013), including those which take context-dependent effects into account (Gupta et al., 2012).
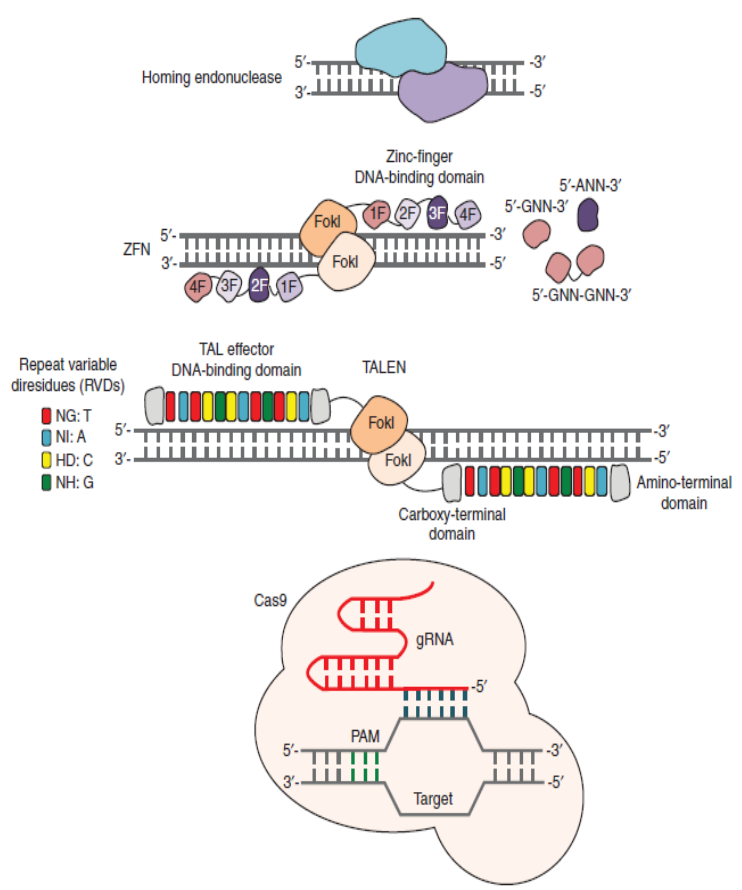

Figure 2. Genome editing technologies. Cartoons showing the mechanisms of targeted nucleases. From top: homing endonucleases, zinc-finger nucleases (ZFNs), transcription activator-like effector (TALE) nucleases (TALENs), and clustered regularly interspaced short palindromic repeats (CRISPR)CRISPR-associated protein 9 (Cas9). Homing endonucleases cleave their DNA substrates as dimers, and do not have distinct binding and cleavage domains. ZFNs observe target sites that consist of two zinc-finger binding sites that flank a 5- to 7-base pair (bp) spacer sequence recognized by the FokI cleavage domain. TALENs notice target sites that consist of two TALE DNA-binding sites that flank a 12- to 20-bp spacer sequence recognized by the FokI cleavage domain. The Cas9 nuclease is targeted to DNA sequences complementary to the targeting sequence within the single guide RNA (gRNA) located instantly upstream of a compatible proto-spacer adjacent motif (PAM). DNA and protein are not peaked to scale (Gaj et al., 2016). 
A

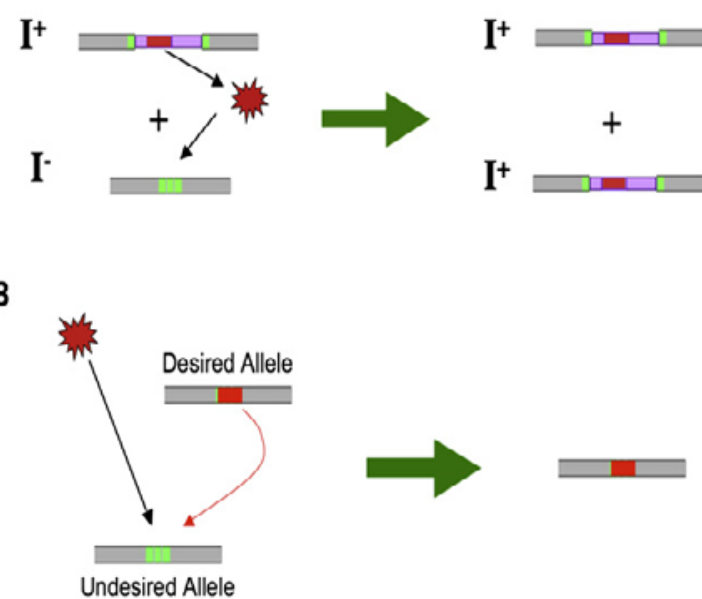

Figure 3. Homing Endonucleases and Genetic Homing (A) Motile element with a homing endonuclease gene (red bar) which is attached within a self-splicing intron or intein (blue bars) resides within a host gene (gray bars). The homing endonuclease (red star) is expressed and cleaves a target site (green bar) which is found in a homologous allele of the host gene lacking the entire element. The arising double-strand break is repaired by cellular machinery, generally leading either to repair via non-homologous end-joining (not shown) or via homologous recombination (HR). If HR successfully uses the intron containing host allele $\left(\mathrm{I}^{+}\right)$as a corrective template, then the original uninterrupted allele (intronminus $\left[\mathrm{I}^{-}\right]$) is converted to an allele that now contains the intron and homing endonuclease gene (intron-plus $\left[\mathrm{I}^{+}\right]$). (B) Properties homing endonuclease introduced for gene expression. Based on the presence or absence (as well as the sequence)of a corrective DNA template for break repair, and on the catalytic properties of the endonuclease, such applications can lead to mutation, knockout, modification, or insertion of exogenous coding DNA into the gene target (Stoddard, 2011).

The use of ZFNs for gene editing (in addition to all targeted nucleases) is off-target mutations (Gabriel et al., 2011). Therefore, several approaches have been undertaken to increase their specificity. For creation of obligate heterodimer ZFNs engineering depends upon the Charge-Charge repulsion for prevent the unwanted homodimof the FokI cleavage domain (Doyon et al., 2011), although the minimizing potential for ZFNs to dimerize at off-target sites. The methods of protein engineering have also been used to boost the cleavage efficiency of the FokI cleavage domain (Guo et al., 2010). The major approach for improving ZFN specificity is to pass them into cells as protein. Due to the peculiar cellpenetrating activity of zinc-finger domains (Gaj et al.,2014), ZFN proteins are naturally cell-permeable and can facilitate the gene editing with fewer off-target effects when tested upon the cells as refined protein compared to when expressed within cells from nucleic acids (Gaj et al., 2012). Afterwards, converted ZFN proteins equipped with enhanced cell-penetrating activity have been described (Liu et al., 2015).

\section{Transcription activator-like effector nucleases (TALENs)}

Transcription activator-like effector nucleases (TALENs) are restriction enzymes or restrictase which can be applied to cut specific sequence of DNA. TALENs provide precise insertion, deletion, or substitutions of specific genes alter the genome. TALENs possess a designed TALE domain that mimics the natural transcription activator-like effector proteins and a nuclease that can cleave DNA in cells. TALENs have emerged as a magical genome editing tools in numerous species and cell types.

\subsection{TALE Nucleases}

TALE proteins are bacterial effectors. The code used by TALE proteins to recognize DNA was discovered in 2009 (Boch et al., 2009). Sooner, this discovery approved the creation of custom TALENs capable of modifying nearly any gene. ZFNs and TALENs are flexible in shape and function, comprised of an amino-terminal TALE DNA-binding domain fused to a carboxy- terminal FokI cleavage domain (Christian et al., 2010; Miller et al., 2011). Dimerization of TALEN proteins is mediated by the FokI cleavage domain like ZFNs which cuts within a 12- to 19-bp spacer sequence that separates each TALE binding site (Fig. 2) (Miller et al., 2011). TALEs are gathered to recognize between 12- to 20-bps of DNA, with more bases typically leading to higher genome-editing specificity (Guilinger et al., 2014). The TALE binding domain consists of a series of repeat domains, each 34 residues in length. All repeat touches DNA via the amino acid residues at positions 12 and 13, known as the repeat variable diresidues (RVDs) (Boch et al., 2009).

Unalike zinc fingers, that verify DNA triplets, each TALE repeat recognizes only a single bp, with little to no target site overlap from adjacent domains (Mak et al., 2012). The most generally used RVDs for assembling synthetic TALE arrays are: NI for adenine, HD for cytosine, NG for thymine, and NN or HN for guanine or adenine (Streubel et al., 2012). TALE DNA-binding domains can be composed using a different method, with the most straightforward approach being Golden Gate assembly (Cermak et al., 2011). TALE assembly methods have also been developed, including FLASH assembly (Reyon et al., 2012), iterative capped assembly (Briggs et al. 2012), and association independent cloning (SchmidBurgk et al., 2013). Transformation in TALEN assembly have focused on the improvement of methods that can promote their performance, including specificity profiling to uncover nonconventional RVDs that improve TALEN activity (Miller et al., 2015), directed evolution as means to refine TALE specificity (Hubbard et al., 2015), and even combining TALE domains to homing endonuclease differing to produce chimeric nucleases with extended targeting specificity (Boissel et al., 2014).

TALENs proposes two distinct advantages for genome editing compared to ZFNs; first, no directed evolution is necessary to engineer TALE arrays, reducing the amount of time and practice needed to assemble a functional nuclease, whereas, second, TALENs have been reported to show upgraded specificity and minimized toxicity compared to some ZFNs (Mussolino et al., 2014) because of their increased closeness for target DNA (Meckler et al., 2013) or perhaps a greater energetic penalty for associating with base mismatches. However, TALENs are substantially larger than ZFNs, and have a highly repetitive structure, making their energetic delivery into cells through the use of lentivirus (Holkers et al., 2012) or a single adenoassociated virus (AAV) challenged the single particle. Approach for reducing the limitations has 
emerged as TALENs can be easily brought into cells as mRNA (Mahiny et al., 2015) and even protein (Cai et al., 2014), even though other codon usage and amino acid deterioration can also be influenced to precise RVD arrays that might be less susceptible to recombination (Kim et al., 2013). Adenoviral vectors are also useful for mediating TALEN delivery to hard-to-transfect cell types (Maggio et al., 2016).

\section{CRISPR-Cas9}

The discovery of CASPR-Cas9 technique dates back to 1987 identified by Atsuo Nakata and colleagues, who discovered a peculiar locus in Escherichia coli K12 strain with five identical sequences of 29 nucleotides spaced by 32 nucleotides downstream of the iap gene (Ishino et al., 1987).The notable characteristics of repeating spacers and direct repeats make CRISPRs easily noticeable in long sequences of DNA, as the number of repeats decreases the likelihood of a false positive match (Sorek et al., 2008). In biological research, CRISPR is becoming an indispensable tool. The programmable capacity of the Cas9 enzyme is now revolutionizing different fields of medicine, biotechnology, and agriculture. The CRISPR-Cas based genome editing approach has become a choice of technique and magical tool due to its simplicity, ease of access, cost, and flexibility whereas previous methods were difficult and expensive to design (Doudna and Charpentier, 2014).

It has brought a revolution in life sciences since their development as an experimental tool in 2012. The technology depends on the formation of sequences called as spacers in the CRISPR region of the host genome. Spacer sequences are identical to sections of invading foreign nucleic acids, commonly from phases. These spacers regions are transcribed into noncoding CRISPRRNA (crRNA), which acts as guide to direct an effort nuclease to make targeted cuts in invading genetic material. The desired cleavage of invading DNA prevents expression of viral elements, which prevents successful infection of the bacterium. In Streptococcus pyogenes, CRISPR-II system requires only one effector protein, Cas9, which can be targeted to make a double-stranded break in DNA at a specific nucleotide sequence (Jinek et al., 2012). CRISPR-associated protein 9 (Cas9) genes are present in approximately $40 \%$ bacterial species such as Streptococcus pyogenes, Staphylococcus aureus, Neisseria meningitides, Staphylococcus epidermidis, Streptococcus mutans, Streptococcus thermophilus, Escherichia coli, Corynebacterium diptheriae and around $90 \%$ archeal species such as Sulfolobussolfataricus, Methanocaldococcus jannaschii, Methanothermobacter thermoautotrophicum, Pyrococcusfuriosus, Haloferax mediterranei, Archaeoglobus fulgidus (Horvath and Barrangou, 2010). CRISPR/Cas9 technology is doing boast of a promising future due to transformed and metamorphosed for potential modify and regulate the prokaryotic and eukaryotic genomes (Das and Paudel, 2020).

\subsection{Mechanism of CRISPR-Cas9 system}

The main function of CRISPR-Cas9 system is to make a double-stranded break into the target DNA where a new gene of interest can be manipulated. In comparison to random mutagenesis like radiation, ethyl methane suffocate (EMS), Zinc finger nucleases (ZFNs) and Transcription activator like effector nucleases (TALENs) genome editing by targeting, CRISPR-Cas9 is more precise and efficient at a specific site. CRISPR-Cas9 possesses specificity towards a particular sequence because of Cas9 protein's unique structural conformation (Figure 4) (Song et al., 2016) and a restricted core, nucleic acid sites and is a bi-lobed structure protein. The nucleic acid sites form recognition (REC) lobe connected with nuclease (NUC) lobe by helix bridge (Doudna and Charpentier, 2014). Cas9 was statically analyzed to be multifaceted crystal structured protein having two nuclease domains that protect the organism (first found in Staphylococcus aureus) from infection by cleaving the assaulting genome from phage and viruses (Stemberg et al., 2014).The Cas9 protein manifested from the host's genomic sequence goals and splits DNA in natural as well as the artificial system of CRISPR-Cas. Cas9 protein is synthesized by the combination of six domains: recognizing domain-1 (REC-I), REC-II, Protospacers adjacent motif(PAM) sequence, RuvC, Bridge helix and a conserved amino acid sequence of His-Asn-His (HNH). The REC-I, PAM, bridge helix, nuclease domains (RuvC and $\mathrm{HNH}$ ) acts as binding to guide RNA, initiating binding of DNA, initiating cleavage on target DNA, cleaving the DNA (HNH cleaving complementary and RuvC cleaving non-complementary strand), respectively (Sternberg et al., 2014). Cas9 becomes excited when bound to SgRNA, i.e. Single guide RNA at REC lobe and conversion of Cas9 into DNA nickase occurs if nucleases get mutated (Ran et al., 2013). Many other different Cas protein including Cas9 inside the host bind to genomic DNA (those having CRISPR sequence), which makes this system so versatile. The total functioning Cas protein has a similar mechanism mediated by RNA, therefore called sgRNA mechanism (Wang et al., 2016).

In this figure, the steps are 1: The entry of foreign DNA into the bacterial cell, step 2: Detection of the foreign DNA and activation of bacterial genome, step 3: Fusion of pre-crRNA, step 4: Ligation of pre-crRNA and trans activating crRNA (tracrRNA) to form the guide RNA, step 5: Binding of inactive Cas9 protein with guide RNA to form Active Cas9 complex, step 6: Detection and binding of Cas9 complex with foreign DNA, step 7: Fragmentation and lysis of foreign DNA. Step A: illustrate the isolation of activated guide RNA-Cas9 complex, Step B: Insertion of target gene sequence and activated guide RNA-Cas9 complex into a suitable vector, Step C: Fragmentation of DNA or at specific locus, Step D: Genome editing with Non-Homologous End Joining (NHEJ) method, Step E: Involvement of homologous pair and Homology Directed Repair (HDR) method of gene editing (Mohanty et al., 2019). 


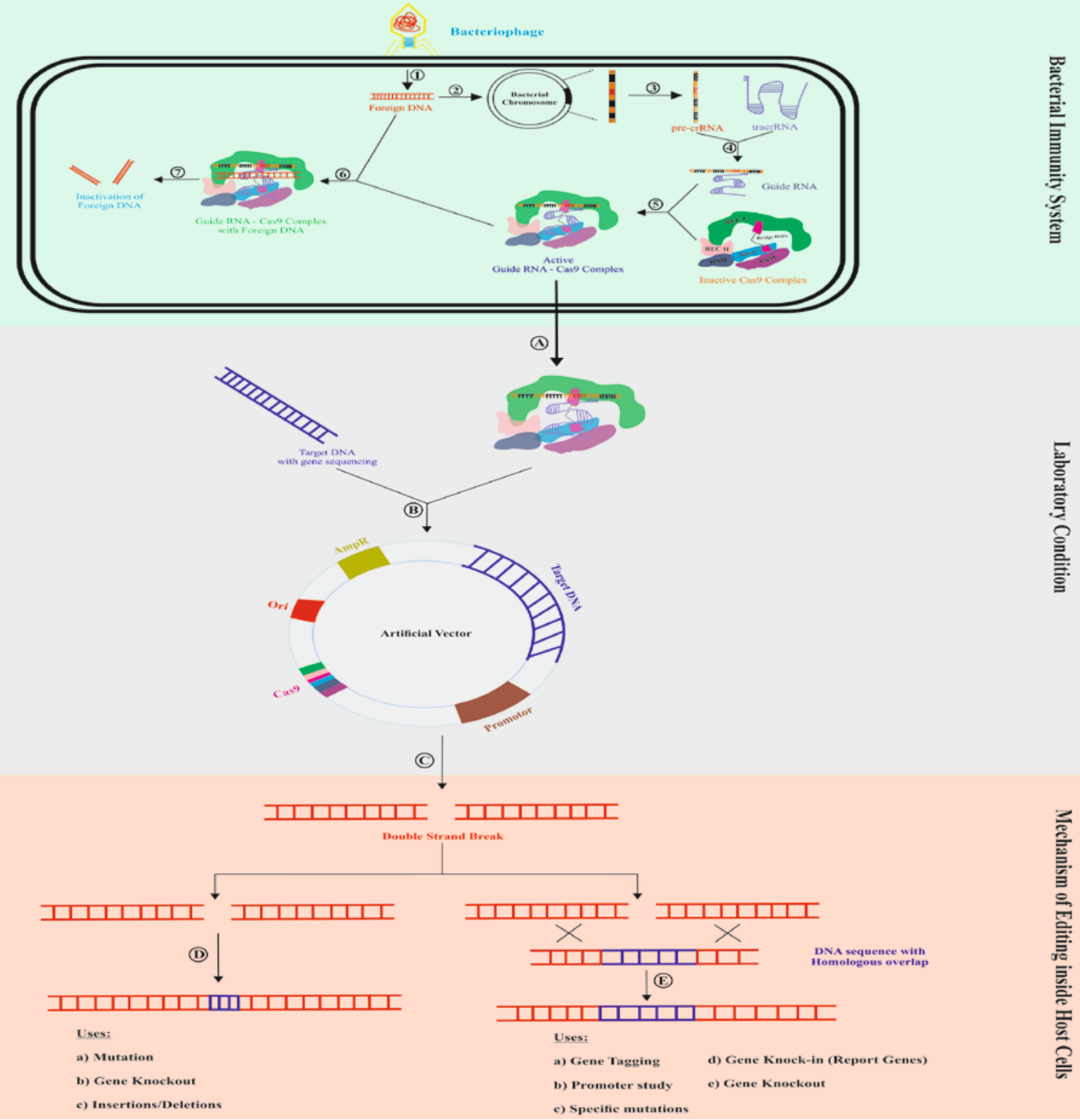

Figure 4. Mechanism of CRISPR-Cas9 system with its uses (Mohanty et al., 2019)

\subsection{Cas Variants and Other Nucleases for Plant Genome Editing}

Streptococcus pyogenes (SpCas9) variant carrying random mutation in to the domain $\mathrm{HNH}$ and RuvC which identified that increased the editing efficiency. Cas9 can be modified into a nickase, capable of producing a single strand cleavage by mutating either the HNH or the RuvClike domain (Xie et al., 2014). Cas9 can also be changed into a DNA binding protein, dead Cas9 (dCas9) by mutating both the domains (dCas9; Asp $^{10} \rightarrow$ Ala, His ${ }^{840}$ $\rightarrow$ Ala). The SpCas9 uses a 5'-NGG-3' protospacer adjacent motif (PAM), and even though 5'-NGG-3' sequence takes place approximately 5-10 times in every $100 \mathrm{bp}$ in model plant species (Xie et al., 2014). The PAM requirement is still a hold up for the Cas9 targetable sites. To get the better of this issue, many Cas9 variants and Cas9 orthologs with various PAM preferences have been applied to get the same results as the wild type CRISPR/Cas9 system. One of that system is CRISPR from Prevotella and Francisella (Cpf1) that is recently cited as Cas12a is a nuclease of class II type V and lacks the HNH domain, possessing only the RuvC-like domain naturally.
Cpf1 yields break sites with staggered cuts rather than blunt ends as Cas9 (Zetsche et al., 2015). Cpf1 requires a $\mathrm{T}$ rich PAM that increases the number of possible plant genetic manipulations and a shorter crRNA than Cas9 (Stella et al., 2017). However, short crRNAs raise the possibility of having a secondary structure in the RNA. Cpf1 edited lines need accurate genomic evaluation as Cpf1 has been shown to cause genomic rearrangements in regions surrounding the target sites (Bernabé et al., 2019).

Nonetheless, Cpf1 has already been used in many plant species such as rice (Begemann et al., 2017; Tang et al., 2018) and Arabidopsis (Tang et al., 2017) and offers a great alternative to Cas9 and a wider range of targetable genes in addition to the ones offered by Cas9. Recently, a new class II system encoding a miniature (529 amino acids) effector, Cas14a1, has been identified. (Karvelis et al., 2019). This Cas variant functions as a PAMindependent single stranded DNA nuclease. Many more Cas variants and orthologs are being discovered (Makarova et al., 2015) and exploited for gene editing purposes since the CRISPR/Cas system is a general 
immune system present in bacteria and archaea for protection against bacteriophages.

Generally, CRISPR/Cas9 technology can be applied to target multiple genes (or multiple sites within a gene) to generate small or large deletions in the genome and provide practical applications in basic and applied biological research. There are two approaches that have been used for expressing multiple gRNA. First, each gRNA is expressed with an individual promoter, and second multiple gRNAs expressed by one promoter as a single transcript which is further processed to release individual gRNAs (Minkenberg et al., 2017).

Transfer-RNAs (tRNAs) are the basic cellular components found in all organisms, and the production and processing are guided by RNA-processing systems. With this concept, Xie et al (2015) developed an endogenous RNA-processing system to obtain multiple gRNA from a single transcript (Figure 5). According to them, a synthesized DNA fragment having tRNA-gRNAin a tandemly arrayed fashion can be processed into gRNAs having the desired 50 targeting sequences, which accurately directed Cas9 protein for editing multiple chromosomal targets. The tRNA-processing system includes RNaseZ and RNaseP, inherently present in a cell, precisely cleaves 50 and 30 ends of the tRNAs, thereby releasing individual gRNAs. By the application of this approach in rice plants, stably inherited mutations were easily achieved with up to $100 \%$ efficiency, and since tRNA processing machinery is nearly conserved in all the organisms, similar efficiency in mutation can be justified in a variety of organisms. The tRNA-based multiple target editing system is preferred over other methods due to several advantages, including the specificity of RNaseP and RNaseZ for tRNA. Only D-loop arm, acceptor stem and $\mathrm{T}$ C-loop arm of tRNA are compulsory for the detection by RNase (Osakabe et al., 2010). The tRNAs also contain an internal Pol III promoter site; therefore, tRNA sequences can also bestrike into as an enhancer system for Pol III.

In case, to explore the synthetic poly-tRNAgRNA(PTG) DNA fragment would be transcribed, processed, and function as anticipated, they manufacture PTG with the structures, tRNA-gRNA (PTG1 and PTG2) or tRNA-gRNA-tRNA (PTG1.1 and PTG2.1), and as a proof, the qRT-PCR analysis declared that the level of PTG was 3 to 31 times higher than the simple sgRNA in rice protoplasts. Furthermore, the full tRNA-gRNA transcripts were not noticed by qRT-PCR, further confirming the efficient cleavage of gRNAs from the tRNA-gRNA transcripts by the tRNA processing system (Figure 3). The Pol III promoters (e.g., U3p) transcribe the PTGs as the SgRNA genes but, PTGs are not obligated to begin with a specific nucleotide as is the case with SgRNAs. Therefore, the vectors used in CRISPR/Cas9 for the expression of SgRNAs can be used accurately to manifest PTGs for the multiplexing approach (Figure 5).

The PTG technology can also be practiced for the enhancement of induction of mutations simultaneously in multiple genomic loci, or for deletion of short fragments of chromosomes. For example, PTG could be used with Cas9 nickase to increase targeting fidelity (Petolino et al., 2010) or with dCas9 transcriptional activator or repressor to manipulate multiple gene expression (Shukla et al., 2009).

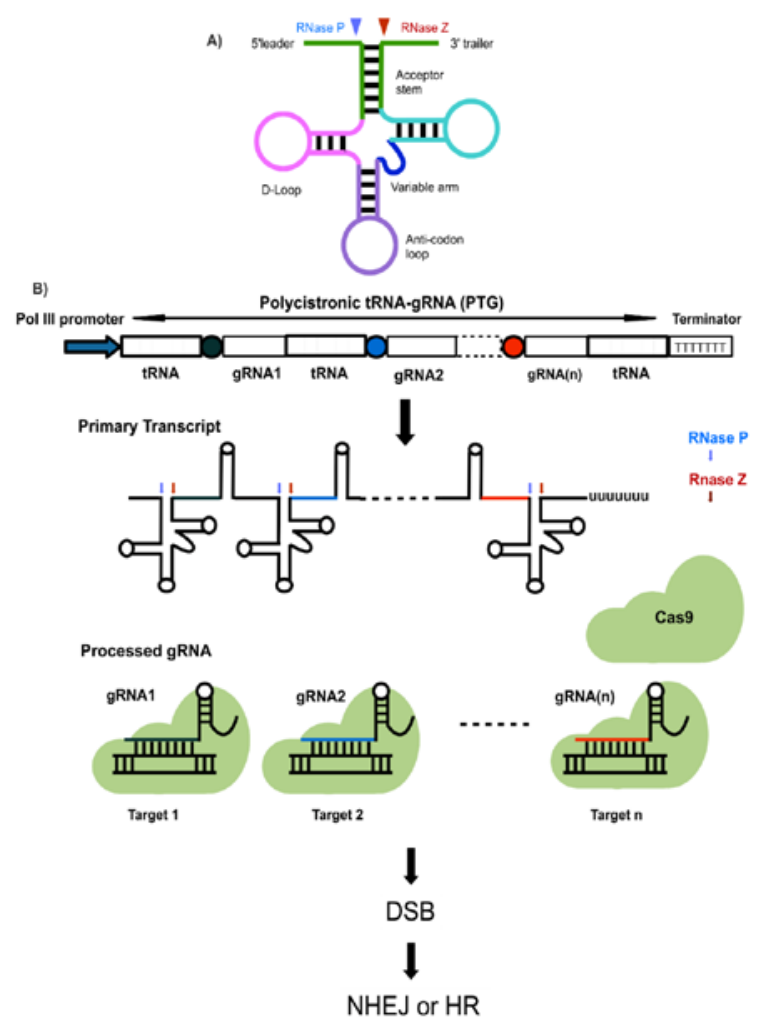

Figure 5. Multigene targeting via CRISPR/Cas9 using PTG/Cas9 method. (A) A eukaryotic pre-tRNAwith a depiction of posttranscriptional processing by RNaseP and RNaseZ (depicted as blue and red arrows respectively), splicing out 50 leader and 30 trailer respectively. (B) Each gRNA with target-specific sequence (labeled here as circles of different colors) and conserved gRNA sequence (blank rectangle) is fused to a tRNA coding sequence (rectangles with boxes), that is cleaved after transcription by RNaseP and RNaseZ to release mature tRNAs and gRNAs (with lines of same colors as the circles). These processed gRNAs direct Cas9 to the target site, which then causes a double-strand break (DSB) repaired by NHEJ or Homologous recombination (HR) (Vats et al., 2019).

\section{Conclusion}

Many attractive features such as simplicity, efficiency, high specificity and amenability to multiplexing, gene editing technologies transforming the way for the next generation breeding. CRISPR/Cas based genome editing system emerged as an evolution in recent years due to its enormous potential to make targeted modifications in the genome and also for versatile diagnostic purposes. Many advancements such as DNA free genome editing systems (RNPs), multiple Cas9 variants, many multi-gene targeting approaches, precise base editing, and measures to increase the frequency of HDR have been achieved very soon. However, crop breeders still need to make significant efforts to implement technological advances in crop improvement programs.

Different from zinc-finger nucleases (ZFNs) and transcription activator like effector nucleases (TALENs), CRISPR/Cas9 takes benefit of an RNA-guided DNA endonuclease enzyme, Cas9, which can generate doublestrand breaks (DSBs) at specific genomic locations. It activates cellular endogenous DNA repair pathways, contributing to the generation of desired modifications in the genome. The capacity of the system has opened up a new pathway in the understanding of amyotrophic lateral 
sclerosis (ALS) pathogenesis and the development of new therapeutic approaches.

\section{Acknowledgements}

Authors are grateful to Swaraj Mohanty for providing his original figure (figure 4). Authors are also thankful to Patrick D. Hsu (figure 1), Thomas Gaj (figure 2), Barry L. Stoddard (figure 3) and Rupesh Deshmukh (figure 5) for the permission to use their figures in this manuscript. Finally, authors are grateful for the reviewer who suggested good comments for the improvement of the manuscript.

\section{Conflict of interest}

We declare that there are no conflicting interests.

\section{References}

Begemann MB, Gray BN, January E, Gordon GC, He Y, Liu H, Wu X, Brutnell TP, Mockler TC and Oufattole M. 2017. Precise insertion and guided editing of higher plant genomes using Cpf1 CRISPR nucleases. Sci. Rep. 7:11606.

Bernabé-Orts JM, Casas-Rodrigo I, Minguet EG, et al. 2019. Assessment of Cas12a-mediated gene editing efficiency in plants. Plant Biotechnol J. 17(10):1971-1984.

Bhakta MS, Henry IM, Ousterout DG, Das KT, Lockwood SH, Meckler JF, Wallen MC, Zykovich A, Yu Y, Leo H, Liferg XU, Gersbach CA and Segal D. 2013. Highly active zinc-finger nucleases by extended modular assembly. Genome Res 23: 530538.

Boch J, Scholze H, Schornack S, Landgraf A, Hahn S, Kay S, Lahaye T, Nickstadt A and Bonas U. 2009. Breaking the code of DNA binding specificity of TAL-type III effectors. Science 326: 1509-1512.

Boissel S, Jarjour J, Astrakhan A, Adey A, Gouble A, Duchateau P, Shendure J, Stoddard BL, Certo MT, Baker D and Scharenberg AM. 2014. megaTALs: A rare-cleaving nuclease architecture for therapeutic genome engineering. Nucleic Acids Res 42: 25912601.

Briggs AW, Rios X, Chari R, Yang L, Zhang F, Mali P and Church GM. 2012. Iterative capped assembly: rapid and scalable synthesis of repeat-module DNA such as TAL effectors from individual monomers. Nucleic Acids Res 40: e117.

Bolotin M, Coen D, Deutsch J, Dujon B, Netter P, Petrochilo E, and Slonimski PP. 1971. La recombinaison des mitochondries chez Saccharomyces cerevisiae. Bull Inst Pasteur, 69:215-239.

Cai Y, Bak RO and Mikkelsen JG. 2014. Targeted genome editing by lentiviral protein transduction of zinc-finger and TALeffector nucleases. eLife 3: $\mathrm{e} 01911$.

Cermak T, Doyle EL, Christian M, Wang L, Zhang Y, Schmidt C, Baller JA, Somia NV, Bogdanove AJ, and Voytas DF. 2011. Efficient design and assembly of custom TALEN and other TAL effector-based constructs for DNA targeting. Nucleic Acids Res 39: e82.

Chevalier BS and Stoddard BL. 2001. Homing endonucleases: structural and functional insight into the catalysts of intron/intein mobility. Nucleic Acids Res. 29:3757-3774.

Das BD and Paudel N. 2020. CRISPR/Cas9 applications and future prospectus in crop genetic improvement. J Multidiscip Sci, 2(2): 56-66.

Doudna JA and Charpentier E. 2014. The new frontier of genome engineering with CRISPR-Cas9. Science, 346:1077-1087.
Doyon Y, Vo TD, Mendel MC, Greenberg SG, Wang J, Xia DF, Miller JC, Urnov FD, Gregory PD and Holmes MC. 2011. Enhancing zinc-finger-nuclease activity with improved obligate heterodimeric architectures. Nat Methods, 8:74-79.

Dreier B, Fuller RP, Segal DJ, Lund CV, Blancafort P, Huber A, Koksch B and Barbas CF III. 2005. Development of zinc finger domains for recognition of the 50-CNN-30 family DNA sequences and their use in the construction of artificial transcription factors. J Biol Chem, 280: 35588- 35597.

Faye G, Dennebouy N, Kujawa C and Jacq C. 1979. Inserted sequence in the mitochondrial 23S ribosomal RNA gene of the yeast Saccharomyces cerevisiae. Mol. Gen. Genet. 168:101-109.

Gabriel R, Lombardo A, Arens A, Miller JC, Genovese P, Kaeppel C, Nowrouzi A, Bartholomae CC, Wang J, Friedman G, et al. 2011. An unbiased genome-wide analysis of zinc-finger nuclease specificity. Nat Biotechnol, 29:816-823.

Gaj T, Guo J, Kato Y, Sirk SJ and Barbas CF III. 2012. Targeted gene knockout by direct delivery of zinc-finger nuclease proteins. Nat Methods, 9:805-807.

Gaj T, Liu J, Anderson KE, Sirk SJ and Barbas CF III. 2014. Protein delivery using Cys2-His2 zinc-finger domains ACS. Chem Biol, 9:1662-1667.

Gaj T, Sirk SJ, Shui S, and Liu J. 2016. Genome-Editing Technologies: Principles and Applications. Cold Spring Harb Perspect Biol, 8:a023754.

Gayon, J. 2016. From Mendel to epigenetics: History of genetics. Comptes Rendus Biologies. 339:225-230.

Guilinger JP, Thompson DB and Liu DR. 2014. Fusion of catalytically inactive Cas9 to FokI nuclease improves the specificity of genome modification. Nat Biotechnol 32:577-82.

Guo J, Gaj T and Barbas CF III. 2010. Directed evolution of an enhanced and highly efficient FokI cleavage domain for zinc finger nucleases. J Mol Biol, 400: 96-107.

Gupta A, Christensen RG, Rayla AL, Lakshmanan A, Stormo GD and Wolfe SA. 2012. An optimized two-finger archive for ZFNmediated gene targeting. Nat Methods, 9: 588-590.

Holkers M, Maggio I, Liu J, Janssen JM, Miselli F, Mussolino C, Recchia A, Cathomen T, and Gonçalves MA. 2012. Differential integrity of TALE nuclease genes following adenoviral and lentiviral vector gene transfer into human cells. Nucleic acids research. 41(5): e63.

Horvath P and Barrangou R. 2010. CRISPR/Cas, the immune system of bacteria and archaea. Science; 327:167-170.

Hsu PD, Lander ES and Zhang F. 2014. Development and Applications of CRISPR-Cas9 for Genome Engineering. Cell, 157(6): 1262-1278.

Hubbard BP, Badran AH, Zuris JA, Guilinger JP, Davis KM, Chen L, Tsai SQ, Sander JD, Joung JK and Liu DR. 2015. Continuous directed evolution of DNA-binding proteins to improve TALEN specificity. Nat Methods,12: 939-942.

Ishino Y, Shinagawa H, Makino K, Amemura M, and Nakata A. 1987. Nucleotide Sequence of the iap Gene, Responsible for alkaline Phosphate Isozyme Conversion in Escherichia coli, and Identification of Gene Product. Journal of Bacteriology, 169 (12): 5429-5433.

Jacquier A, and Dujon B .1985. An intron-encoded protein is active in a gene conversion process that spreads an intron into a mitochondrial gene. Cell, 41:383-394.

Jinek M, Chylinski K, Fonfara L, Hauer M, Daudna JA, and charpentier E. 2012. A programmable dual-RNA- guided endonucleases in adaptive bacterial immunity . Science, 337 (6096): 816-822. 
Karvelis T, Bigelyte G, Young JK, Hou Z, Zedaveinyte R, Pociute K, Silanskas A, Venclovas C and Siksnys V. 2019. PAM recognition by miniature CRISPR-Cas14 triggers programmable double-stranded DNA cleavage. Bio Rxiv (Preprint), Doi:http://doi.org/10.1101/654897

Kim HJ, Lee HJ, Kim H, Cho SW and Kim JS. 2009. Targeted genome editing in human cells with zinc finger nucleases constructed via modular assembly. Genome Res, 19: 1279-1288.

Kim Y, Kweon J, Kim A, Chon JK, Yoo JY, Kim HJ, Kim S, Lee C, Jeong E, Chung E, et al. 2013a.A library of TAL effector nucleases spanning the human genome. Nat Biotechnol, 31: 251258.

Kim YG, Cha J and Chandrasegaran S. 1996. Hybrid restriction enzymes: Zinc finger fusions to Fok I cleavage domain. Proc Natl Acad Sci, 93: 1156-1160.

Liu J, Gaj T,Wallen MC and Barbas CF III. 2015. Improved cell penetrating zinc-finger nuclease proteins for precision genome engineering. Mol Ther Nucleic Acid,,4: e232.

Maeder ML, Thibodeau-Beganny S, Osiak A, Wright DA, Anthony RM, EichtingerM, Jiang T, Foley JE,Winfrey RJ, Townsend JA, et al. 2008. Rapid “open-source" engineering of customized zinc-finger nucleases for highly efficient gene modification. Mol Cell, 31: 294-301.

Maggio I, Stefanucci L, Janssen JM, Liu J, Chen X,Mouly V and Goncalves MA. 2016. Selection-free gene repair after adenoviral vector transduction of designer nucleases: rescue of dystrophin synthesis in DMD muscle cell populations. Nucleic Acids Res, 44: 1449-1470.

Magnenat L, Blancafort P and Barbas CF III. 2004. In vivo selection of combinatorial libraries and designed affinity maturation of polydactyl zinc finger transcription factors for ICAM-1 provides new insights into gene regulation. $\mathrm{J} \mathrm{Mol} \mathrm{Biol,}$ 341: 635-649.

Mahiny AJ, Dewerth A, Mays LE, Alkhaled M, Mothes B, Malaeksefat E, Loretz B, Rottenberger J, Brosch DM, Reautschnig $\mathrm{P}$, et al. 2015. In vivo genome editing using nucleaseencoding mRNA corrects SP-B deficiency. Nat Biotechnol, 33: 584-586.

Mak AN, Bradley P, Cernadas RA, Bogdanove AJ and Stoddard BL. 2012. The crystal structure of TAL effector PthXo1 bound to its DNA target. Science, 335: 716-719.

Makarova KS, Wolf YI, Alkhnbashi OS, Costa F, Shah SA, Saunders SJ, Barrangou R, Brouns SJ, Charpentier E and Haft D. 2015. An updated evolutionary classification of CRISPR-Cas systems. Nat Rev Genet, 13:722.

Meckler JF, Bhakta MS, Kim MS, Ovadia R, Habrian $\mathrm{CH}$, Zykovich A, Yu A, Lockwood SH, Morbitzer R, Elsaesser J, Lahaye T, Segal D and Baldwin EP. 2013. Quantitative analysis of TALE-DNA interactions suggests polarity effects. Nucleic Acids Res.; 41(7):4118-4128.

Miller JC, Tan S, Qiao G, Barlow KA, Wang J, Xia DF, Meng X, Paschon DE, Leung E, Hinkley SJ, Dlay GP, Hua KL, Ankoudinova I, Cost GJ, Urnov FD, Zhang HS, Holmes MC, Zhang L, Gregory PD and Rebar EJ. 2011. A TALE nuclease architecture for efficient genome editing. Nat Biotechnol, 29 (2):143-148.

Miller JC, Zhang L, Xia DF, Campo JJ, Ankoudinova IV, Guschin DY, Babiarz JE, Meng X, Hinkley SJ, Lam SC, Paschan DE, Vincent AI, Dulay GP, Barlow KA, Shivak DA, Leung E, Kim JD, Amora R, Urnov FD, Gregory PD and Rebar EJ. 2015. Improved specificity of TALE-based genome editing using an expanded RVD repertoire. Nat Methods, 12(5): 465-471.
Minkenberg B, Wheatley M and Yang Y. 2017. CRISPR/Cas9enabled multiplex genome editing and its application. In Progress in Molecular Biology and Translational Science; Elsevier: Amsterdam, The Netherlands, 149: pp. 111-132.

Mohanty S, Dash A and Pradhan CK. 2019. CRISPR-Cas9 Technology: A magical tool for DNA editing, Int $J$ of Biosciences and Bioengineering, 1:005.

Mussolino C, Alzubi J, Fine EJ, Morbitzer R, Cradick TJ, Lahaye T, Bao G and Cathomen T. 2014. TALENs facilitate targeted genome editing in human cells with high specificity and low cytotoxicity. Nucleic Acids Res, 42: 6762-6773.

Netter P, Petrochilo E, Slonimski PP, Bolotin-Fukuhara M, Coen D, Deutsch J and Dujon B. 1974. Mitochondrial genetics. VII. Allelism and mapping studies of ribosomal mutants resistant to chloramphenicol, erythromycin and spiramycin in S. cerevisiae. Genetics, 78:1063-1100.

Osakabe K, Osakabe Y and Toki S. 2010. Site-directed mutagenesis in Arabidopsis using custom-designed zinc finger nucleases. Proc Natl Acad Sci USA, 107:12034-12039.

Pavletich NP and Pabo CO. 1991. Zinc finger-DNA recognition: Crystal structure of a Zif268-DNA complex at 2.1 A. Science, 252: 809-817.

Petolino JF, Worden A, Curlee K, Connell J, Moynahan TLS, Larsen C and Russell S. 2010. Zinc finger nuclease-mediated transgene deletion. Plant Mol Biol, 73:617-628.

Rai KM, Ghose K, Rai A, Singh H, Srivastava R, and Mendu V. 2019. Genome Engineering Tools in Plant Synthetic Biology. In Current Developments in Biotechnology and Bioengineering; Elsevier: Amsterdam, The Netherlands, pp. 47-73.

Ran FA, P.D. Hsu PD, Wright J, Agarwala V, D.A. Scott DA, and Zhang F . 2013. Genome engineering using the CRISPRCas9 system. J Nature Protocols, 8:2281.

Reyon D, Tsai SQ, Khayter C, Foden JA, Sander JD and Joung JK. 2012. FLASH assembly of TALENs for high-throughput genome editing. Nat Biotechnol, 30: 460-465.

Schmid-Burgk JL, Schmidt T, Kaiser V, Honing K and Hornung V. 2013. A ligation-independent cloning technique for highthroughput assembly of transcription activatorlike effector genes. Nat Biotechnol, 31: 76-81.

Shukla VK, Doyon Y, Miller JC, DeKelver RC, Moehle EA, Worden SE, Mitchell JC, Arnold NL, Gopalan S and Meng X. 2009. Precise genome modification in the crop species Zea mays using zinc-finger nucleases. Nature, 459:437.

Silva A, Almeida B, Sampaio-Marques B, Reis MIR. et. al. 2011. Glyceraldehyde-3-phosphate dehydrogenase (GAPDH) is a specific substrate of yeast metacaspase./ Biochimica et Biophysica Acta, 1813, 2044-2049.

Smith J, Bibikova M, Whitby FG, Reddy AR, Chandrasegaran S and Carroll D. 2000. Requirements for double-strand cleavage by chimeric restriction enzymes with zinc finger DNA-recognition domains. Nucleic Acids Res, 28: 3361-3369.

Song G, Balakrishnan R, Binkley G, Costanzo MC, Dalusag K, Demeter J, Engel S, Hellerstedt ST, Karra K, et al. 2016. Integration of new alternative reference strain genome sequences into the Saccharomyces genome database. Oxford University Press, article ID baw074.

Sorek R, Kunin V and Hugenholtz P. 2008. CRISPR - a widespread system that provides acquired resistance against phages in bacteria and archaea. Nat Rev Microbiol, 6:181-186.

Stella S, Alcón P and Montoya GJN. 2017. Structure of the Cpf1 endonuclease R-loop complex after target DNA cleavage. Nature, 546:559. 
Sternberg SH, Redding S, Jinek M, Greene EC, and Doudna JA.2014. DNA interrogation by the CRISPR RNA-guided endonuclease Cas9. J Nature. 507: 62.

Stoddard BL. 2005. Homing endonuclease structure and function. Quarterly Reviews of Biophysic, 38: 49-95.

Stoddard BL. 2011. Homing Endonucleases: From Microbial Genetic Invaders to Reagents for Targeted DNA Modification, Structure 19: 7-15.

Stoddard BL. 2014. Homing endonucleases from mobile group I introns: discovery to genome engineering. Mobile DNA, 5: 7.

Streubel J, Blucher C, Landgraf A and Boch J. 2012. TAL effector RVD specificities and efficiencies. Nat Biotechnol 30: 593-95.

Tang X, Liu G, Zhou J, Ren Q, You Q, Tian L, Xin X, Zhong $Z$, Liu B and Zheng X. 2018. A large-scale whole-genome sequencing analysis reveals highly specific genome editing by both Cas9 and Cpf1 (Cas12a) nucleases in rice. Genome Biol. 19: 84.

Tang X, Lowder LG, Zhang T, Malzahn AA, Zheng X, Voytas DF, Zhong Z, Chen Y, Ren Q and Li QA. 2017. CRISPR-Cpf1 system for efficient genome editing and transcriptional repression in plants. Nat. Plants, 3:17018.
Urnov FD, Rebar EJ, Holmes MC, Zhang HS and Gregory PD. 2010. Genome editing with engineered zinc finger nucleases. Nat Rev Genet, 11: 636-646.

Vats S, Kumawat S, Kumar V, Patil GB, Joshi T, Sonah H, Sharma TR, and Deshmukh R. 2019. Genome Editing in Plants: Exploration of Technological Advancements and Challenges, Cells, 8:1386;

Wang H, La Russa M, and Qi LS. 2016. CRISPR/Cas9 in genome editing and beyond. J Annual review of biochemistry, 85:227-264.

Wolfe SA, Nekludova L and PaboCO. 2000. DNA recognition by Cys2His2 zinc finger proteins. Annu Rev Biophys Biomol Struct, 29: $183-212$

Xie K, Zhang J and Yang Y.2014. Genome-wide prediction of highly specific guide RNA spacers for CRISPR-Cas9-mediated genome editing in model plants and major crops. Mol Plant, 7:923-926

Xie Y, Kang R, Sun X, Zhong M, Huang J, Klionsky DJ, and Tang D. 2015. Posttranslational modification of autophagy-related proteins in macroautophagy, Autophagy, 11: 28-45. 